\title{
FRACTURE ANALYSIS OF A NONHOMOGENEOUS BEAM WITH TWO CONCENTRIC CYLINDRICAL LONGITUDINAL CRACKS
}

\author{
Victor Rizov* \\ Department of Technical Mechanics, Faculty of Hydro-technique, University of Architecture, Civil Engineering and \\ Geodesy, 1 Chr. Smirnesky blvd., 1046 - Sofia, Bulgaria
}

\begin{tabular}{l}
\hline ARTICLE INFO \\
\hline Article history: \\
Received: 12.7 .2019$. \\
Received in revised form: 14.10 .2019$. \\
Accepted: 17.10 .2019$. \\
\hline Keywords: \\
Nonhomogeneous material \\
Longitudinal fracture \\
Two circular cylindrical cracks \\
Varying cross-section \\
Material non-linearity \\
\hline DOI: https://doi.org/10.30765/er.1497
\end{tabular}

\begin{abstract}
:
This paper is concerned with the analysis of the fracture behaviour of a nohomogeneous cantilever beam with two concentric longitudinal cracks. The beam has a circular crosssection with linearly varying radius along the beam length. Moreover, the beam exhibits continuously varying material inhomogeneity in the radial direction. The fracture is analyzed in terms of strain energy release rate assuming nonlinear mechanical behaviour of the material. For this purpose, solutions for the strain energy release rate are derived by considering the energy balance. Two cantilever beam configurations with different lengths of longitudinal cracks are analysed. Moreover, the two cracks are arranged arbitrarily in the radial direction. The longitudinal fracture behaviour of the beam is also analysed by considering the complementary strain energy for verification. The strain energy release rate solutions are used to investigate the influence of varying radius of the cross section along the length of the beam on the longitudinal fracture behaviour. The effects of crack lengths and the location of the two concentric cracks in the radial direction on fracture are also studied. The influences of the loading conditions of the beam and the inhomogeneity of the material in the radial direction on the fracture behaviour are also evaluated.
\end{abstract}

\section{Introduction}

Technical development is inextricably linked to the extensive use of high performance structural members and components in various load bearing applications in mechanical and civil engineering. The driving force behind the technical development is often the desire to increase strength and improve stability while reducing the weight and the prime cost of structures and equipment. One of the ways to achieve these conflicting goals is to use members and components with continuously varying cross-sections in the length direction. For example, beam structures with continuously varying cross-section in the length provide an efficient distribution of their strength. It is therefore not surprising that the use of beams with continuously varying cross-section is widespread in various fields of modern engineering, such as lightweight structures, robotics, aircraft, marine structures, automotive industry, turbines and others.

The efficiency of beam structural members with continuously varying cross-section in the longitudinal direction can be further enhanced by using inhomogeneous materials. The microstructure of the inhomogeneous materials varies continuously (uniformly) along one or more directions in the solid. Thus, the material properties are a function of coordinates. The strong interest of the scientific community in inhomogeneous materials is largely due to the fact that certain kinds of inhomogeneous materials, such as

\footnotetext{
* Corresponding author.

E-mail address: V_RIZOV_FHE@UACG.BG
} 
functionally graded materials, have been intensively used in the last thirty years [1, 2]. Functionally graded materials are advanced inhomogeneous composites prepared by continuously mixing two or more constituents according to a certain volume fraction. The technology used to fabricate functionally graded materials permit gradual compositional change.

Thus, one can tailor the spatial variation of the material properties of the functionally graded materials during the manufacturing process to achieve optimal performance of the parts and components against external loads and influences. Since the distribution of material properties in the solid can be arbitrarily designed, functionally graded materials are particularly suitable for meeting the requirements of different material properties in different parts of a component. In addition, functionally graded materials are ideally suited for structural applications in extreme environments (severe temperature gradients, chemical agents). As a result, functionally graded materials have undisputed advantages over classical homogeneous structural materials in areas such as aeronautics, nuclear reactors, robotics, microelectronics and biomedicine.

It should be noted that certain types of inhomogeneous materials, such as functionally graded materials, can be built up in layers [3,4]. One of the weaknesses of these materials is that they are highly susceptible to the occurrence of longitudinal cracks between layers. The longitudinal cracks reduce the strength and stiffness, degrade the stability behaviour, and can lead to catastrophic failure of the entire structure. Therefore, the study of longitudinal cracks in non-homogeneous beams is especially significant for practical design.t should be mentioned, however, that fracture analysis of nonhomogeneous structural members and components is much more complicated in comparison with that of homogeneous structures due to the fact that the material properties of nonhomogeneous materials are functions of coordinates [5 - 13]. Recently, several works on longitudinal fracture of nonhomogeneous (functionally graded) beam structures have been published [14 - 16]. These works are focussed on analyses of fracture behaviour of nonhomogeneous beams of a constant rectangular cross-section along the beam length with one longitudinal crack.

Therefore, the aim of the present paper is to analyze the fracture behaviour of a cantilever beam with a circular cross-section whose radius varies linearly along the length of the beam. Two concentric longitudinal cracks of different lengths are located arbitrarily in the radial direction. The beam exhibits continuously varying material inhomogeneity in the radial direction. The fracture is studied in terms of strain energy release rate assuming nonlinear elastic mechanical behaviour of the material. The strain energy release rate is derived by considering the energy balance. The fracture is also analysed considering the complementary strain energy in the beam for verification.

\section{Calculation of the strain energy release rate}

\subsection{Consideration of the energy balance}

The present paper is focused on analyzing the fracture behaviour of the nonhomogeneous cantilever beam configuration with two concentric longitudinal cracks shown in Figure 1. The beam is clamped in section, $B$. The length of the beam is denoted by $l$.

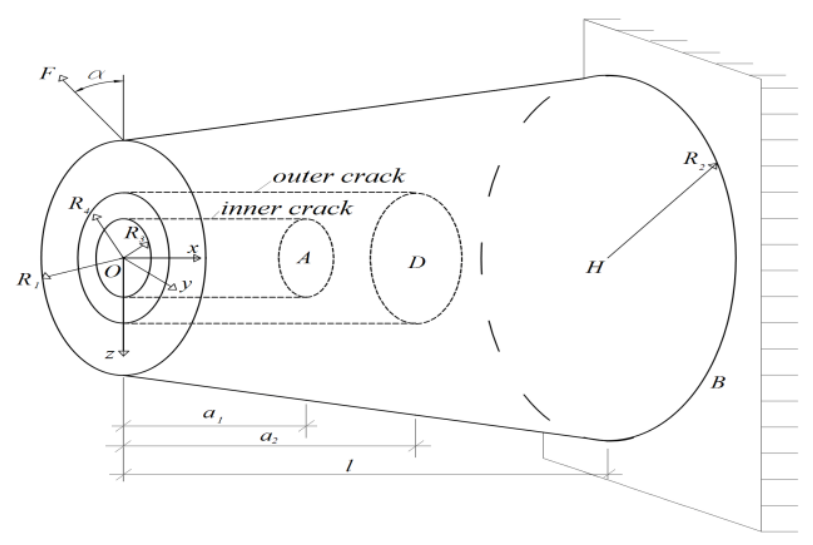

Figure 1. Geometry and loading of nonhomogeneous cantilever beam with two concentric longitudinal cracks (the outer crack is longer than the inner one). 
The beam has a circular cross-section. The radius of the cross-section, $R$, varies linearly from $R_{1}$ at the free end of the beam to $R_{2}$ at the clamped end

$$
R=R_{1}+\frac{R_{2}-R_{1}}{l} x
$$

Where

$$
0 \leq x \leq l
$$

In (1), $x$ is the longitudinal centroidal axis of the beam. Two concentric longitudinal circular cylindrical cracks are located arbitrary in radial direction. The lengths of the inner and outer cracks are $a_{1}$ and $a_{2}$, respectively (Figure 1). The inner and outer cracks are circular cylindrical surfaces of radiuses, $R_{3}$ and $R_{4}$, respectively. Thus, the fronts of the inner and outer cracks are circles of radiuses, $R_{3}$ and $R_{4}$, respectively. The beam is loaded by one concentrated force, $F$, applied at the free end of the beam. The force, $F$, is located in the vertical coordinate plane, $x O z$. The orientation of $F$ is given by angle, $\alpha$. The beam exhibits continuously changing material nonhomogeneity in radial direction. Beside, the material has nonlinear elastic mechanical behaviour.

The fracture behaviour is studied in terms of the strain energy release rate. In order to derive the strain energy release rate, the balance of the energy is considered. For this purpose, first, a small increase, $\delta a_{1}$, of the length of the inner crack has been given. The balance of the energy is written as

$$
F \delta w_{p r}=\frac{\partial U}{\partial a_{1}} \delta a_{1}+G_{a_{1}} l_{c f} \delta a_{1}
$$

where $w_{p r}$ is the projection of the displacement of the application point of $F$ on the direction of $F, U$ is the strain energy in the beam, $G_{a_{1}}$ is the strain energy release rate due to the increase of the length of the inner crack, $l_{c f}$ is the length of the front of the inner crack. Since the front of the inner crack is a circle of radius, $R_{3}$, the length of the crack front is found as

$$
l_{c f}=2 \pi R_{3}
$$

Form (3) and (4), one obtains

$$
G_{a_{1}}=\frac{F}{2 \pi R_{3}} \frac{\partial w_{p r}}{\partial a_{1}}-\frac{1}{2 \pi R_{3}} \frac{\partial U}{\partial a_{1}}
$$

In the beam portion $O A$ (Figure 1) the two concentric longitudinal cracks divide the beam in three parts: external, interstitial and internal part. The cross-section of the external part is a ring whose external and internal radiuses are $R$ and $R_{4}$, respectively ( $R$ is determined by (1)). The interstitial part of the beam has a ring-shaped cross-section with external and internal radiuses, $R_{4}$ and $R_{3}$, respectively. The internal part of the beam has a circular cross-section of radius, $R_{3}$.

In the beam portion $A D$, the outer longitudinal crack divides the beam in external and internal parts (Figure 1). The external part has a ring-shaped cross-section. The external and internal radiuses of the ring are $R$ and $R_{4}$, respectively. The cross-section of the internal part of the beam is a circle of radius, $R_{3}$.

Since the beam is divided in six parts, the strain energy cumulated in the beam is written as 


$$
\begin{aligned}
& U=U_{O A 1}+U_{O A 2}+U_{O A 3}+ \\
& +U_{A D 1}+U_{A D 2}+U_{U N}
\end{aligned}
$$

where $U_{O A 1}, U_{O A 2}$ and $U_{O A 3}$ are the strain energies in the external, interstitial and internal parts of beam portion $O A$, respectively, $U_{A D 1}$ and $U_{A D 2}$ are the strain energies in the external and internal parts of beam portion $A D$, respectively, $U_{U N}$ is the strain energy in the un-cracked beam portion, $D H$.

The strain energy in the external part of beam portion $O A$ is obtained as

$$
U_{O A 1}=\int_{0}^{a_{1}}\left[\int_{R_{4}}^{R}\left(\int_{0}^{2 \pi} u_{0 O A 1} r d \varphi\right) d r\right] d x
$$

where $u_{0 O A 1}$ is the strain energy density, $r$ and $\varphi$ are the polar coordinates. The mechanical behaviour of the material is treated by using the following non-linear stress-strain relation [17]:

$$
\sigma=L\left[1-\left(1-\frac{\varepsilon}{g}\right)^{t}\right]
$$

where $\sigma$ is the normal stress, $\varepsilon$ is the strain, $L, g$ and $t$ are material constants. The strain energy density, $u_{0 O A 1}$, in the external part of beam portion $O A$ is obtained by integrating of (8) in boundaries from 0 to $\varepsilon$

$$
u_{0 O A 1}=L \varepsilon+\frac{L g}{t+1}\left(1-\frac{\varepsilon}{g}\right)^{t+1}-\frac{L g}{t+1}
$$

Since the beam exhibits continuously changing material nonhomogeneity in radial direction, the material property, $L$, varies in radial direction according to the following power law:

$$
L=L_{0}+\frac{L_{P}-L_{0}}{R^{f}} r^{f}
$$

where

$$
0 \leq r \leq R
$$

In (10), $L_{0}$ and $L_{P}$ are the values of $L$ at the centre of the cross-section and at the periphery of the beam, respectively, $f$ is a material constant that controls the material nonhomogeneity in radial direction.

Since a beam of high length to diameter ratio is under consideration in the present paper, the distribution of the strains in the cross-section is treated in accordance with the Bernoulli's hypothesis for plane sections. Thus, $\varepsilon$ is distributed linearly along the height of cross-section of the external part of beam portion $O A$

$$
\varepsilon=\kappa_{O A 1}\left(z_{1}-z_{1 n O A 1}\right)
$$

where $\kappa_{O A 1}$ is the curvature, $z_{1}$ is the vertical centroidal axis of the cross-section, $z_{1 \text { nOA1 }}$ is the coordinate of the neutral axis of external part of beam portion $O A$. By using the polar coordinates, (12) is re-written as 


$$
\varepsilon=\kappa_{O A 1}\left(r \sin \varphi-z_{1 n O A 1}\right)
$$

The curvature and the coordinate of the neutral axis are obtained in the following way. First, the equations for equilibrium of the stress resultants in the cross-section of the external part of beam portion $O A$ are written

$$
\begin{gathered}
N_{O A 1}=\int_{R_{4}}^{R}\left(\int_{0}^{2 \pi} \sigma r d \varphi\right) d r \\
M_{O A 1}=\int_{R_{4}}^{R}\left(\int_{0}^{2 \pi} \sigma r^{2} \sin \varphi d \varphi\right) d r
\end{gathered}
$$

where $N_{O A 1}$ and $M_{O A 1}$ are the axial force and the bending moment in the external part of beam portion $O A$. Figure 1 indicates that

$$
N_{O A 1}=F \sin \alpha
$$

The stress, $\sigma$, in (14) and (15) is expressed by (8) where the distribution of $\varepsilon$ is presented by (13). Thus, there are three unknowns, $M_{O A 1}, \kappa_{O A 1}$ and $z_{1 n O A 1}$, in equations (14) and (15). Other four equations are written by considering the equilibrium of the stress resultants in the cross-sections of the interstitial and internal part of beam portion $O A$

$$
\begin{gathered}
N_{O A 2}=\int_{R_{3}}^{R_{4}}\left(\int_{0}^{2 \pi} \sigma_{O A 2} r d \varphi\right) d r \\
M_{O A 2}=\int_{R_{3}}^{R_{4}}\left(\int_{0}^{2 \pi} \sigma_{O A 2} r^{2} \sin \varphi d \varphi\right) d r \\
N_{O A 3}=\int_{0}^{R_{3}}\left(\int_{0}^{2 \pi} \sigma_{O A 3} r d \varphi\right) d r \\
M_{O A 3}=\int_{0}^{R_{3}}\left(\int_{0}^{2 \pi} \sigma_{O A 3} r^{2} \sin \varphi d \varphi\right) d r
\end{gathered}
$$

where $N_{O A 2}, M_{O A 2}$ and $\sigma_{O A 2}$ are, respectively, the axial force, the bending moment and the normal stress in the cross-section of interstitial part, $N_{O A 3}, M_{O A 3}$ and $\sigma_{O A 3}$ are, respectively, the axial force, the bending moment and the normal stress in the cross-section of internal part. It follows from Figure 1 that

$$
\begin{aligned}
& N_{O A 2}=0 \\
& N_{O A 3}=0
\end{aligned}
$$

The distribution of the strain, $\varepsilon_{O A 2}$, in the cross-section of interstitial part is written as 


$$
\varepsilon_{O A 2}=\kappa_{O A 2}\left(r \sin \varphi-z_{1 n O A 2}\right)
$$

where $\kappa_{O A 2}$ is the curvature, $z_{1 n O A 2}$ is the coordinate of the neutral axis. The normal stress, $\sigma_{O A 2}$, is obtained by substituting of (23) in (8). Formula (24) is applied also to express the distribution of the strain, $\varepsilon_{O A 3}$, in the cross-section of the internal part of beam portion $O A$. For this purpose, $\kappa_{O A 2}$ and $z_{1 n O A 2}$ replaced, respectively, with $\kappa_{O A 3}$ and $z_{1 n O A 3}$ where $\kappa_{O A 3}$ and $z_{1 n O A 3}$ are the curvature and the neutral axis of the internal part. Then $\varepsilon_{O A 3}$ is substituted in (8) to express the normal stress, $\sigma_{O A 3}$, in the internal part. The bending moment, $M$, in the beam cross-section is distributed on the three parts of beam portion $O A$. Thus, it can be written that

$$
M_{O A 1}+M_{O A 2}+M_{O A 3}=M
$$

It is evident from Figure 1 that

$$
M=F x \cos \alpha-F R_{1} \sin \alpha
$$

where

$$
0 \leq x \leq l
$$

The curvatures of the external, interstitial and internal parts of the beam portion $O A$ are the same. Thus, it can be written that

$$
\begin{aligned}
& \kappa_{O A 2}=\kappa_{O A 1} \\
& \kappa_{O A 3}=\kappa_{O A 2}
\end{aligned}
$$

By substituting of (8), (10), (13) and (23) in (14), (15), (17), (18), (19) and (20) one obtains six non-linear algebraic equations. These equations together with (24), (27) and (28) are solved numerically with respect to $\kappa_{O A 1}, z_{1 n O A 1}, \kappa_{O A 2}, z_{1 n O A 2}, \kappa_{O A 3}, z_{1 n O A 3}, M_{O A 1}, M_{O A 2}$ and $M_{O A 3}$ by the MatLab computer program by applying the quasi Newton methods. Then, $u_{0 O A 1}$ is obtained by substituting of (13) in (9).

The strain energy in the interstitial part of beam portion $O A$ is expressed as

$$
U_{O A 2}=\int_{0}^{a_{1}}\left[\int_{R_{3}}^{R_{4}}\left(\int_{0}^{2 \pi} u_{0 O A 2} r d \varphi\right) d r\right] d x
$$

where $u_{0 O A 2}$ is the strain energy density. Formula (9) is used to obtain $u_{0 O A 2}$. For this purpose, $\varepsilon$ is replaced with $\varepsilon_{O A 2}$.

The strain energy density, $u_{0 O A 3}$, is integrated in the volume of the internal part of beam portion $O A$ to obtain the strain energy cumulated in this part

$$
U_{O A 3}=\int_{0}^{a_{1}}\left[\int_{0}^{R_{3}}\left(\int_{0}^{2 \pi} u_{0 O A 3} r d \varphi\right) d r\right] d x
$$

where $u_{0 O A 3}$ is found by replacing of $\varepsilon$ with $\varepsilon_{O A 3}$ in (9).

The strain energy in the external part of beam portion $A D$ is written as (Figure 1) 


$$
U_{A D 1}=\int_{a_{1}}^{a_{2}}\left[\int_{R_{4}}^{R}\left(\int_{0}^{2 \pi} u_{0 A D 1} r d \varphi\right) d r\right] d x
$$

where the strain energy density, $u_{0 A D 1}$, is obtained by replacing of $\varepsilon$ with $\varepsilon_{A D 1}$ in (9). The strain, $\varepsilon_{A D 1}$, in the external part of beam portion $A D$ is expressed by (13). For this purpose, $\kappa_{O A 1}$ and $z_{1 n O A 1}$ are replaced with $\kappa_{A D 1}$ and $z_{1 n A D 1}$, respectively. Here, $\kappa_{A D 1}$ and $z_{1 n A D 1}$ are the curvature and the neutral axis coordinate in the external part of beam portion $A D$. The following equations for equilibrium of the stress resultants in cross-sections of the external and internal parts of beam portion $A D$ are used to obtain $\kappa_{A D 1}$ and $z_{1 n A D 1}$ :

$$
\begin{gathered}
N_{A D 1}=\int_{R_{4}}^{R}\left(\int_{0}^{2 \pi} \sigma_{A D 1} r d \varphi\right) d r \\
M_{A D 1}=\int_{R_{4}}^{R}\left(\int_{0}^{2 \pi} \sigma_{A D 1} r^{2} \sin \varphi d \varphi\right) d r \\
N_{A D 2}=\int_{0}^{R_{4}}\left(\int_{0}^{2 \pi} \sigma_{A D 2} r d \varphi\right) d r \\
M_{A D 2}=\int_{0}^{R_{4}}\left(\int_{0}^{2 \pi} \sigma_{A D 2} r^{2} \sin \varphi d \varphi\right) d r
\end{gathered}
$$

where $N_{A D 1}, M_{A D 1}$ and $\sigma_{A D 1}$ are the axial force, the bending moment and the normal stress in the crosssection of the external part, $N_{A D 2}, M_{A D 2}$ and $\sigma_{A D 2}$ are the axial force, the bending moment and the normal stress in the cross-section of internal part. It is evident from Figure 1 that

$$
\begin{gathered}
N_{A D 1}=F \sin \alpha \\
N_{A D 2}=0
\end{gathered}
$$

Besides,

$$
\begin{gathered}
M_{A D 1}+M_{A D 2}=M \\
\kappa_{A D 1}=\kappa_{A D 2}
\end{gathered}
$$

The normal stress, $\sigma_{A D 1}$, is expressed by replacing of $\varepsilon$ with $\varepsilon_{A D 1}$ in (8). The strain, $\varepsilon_{A D 1}$, is obtained by (13). For this purpose, $\kappa_{O A 1}$ and $z_{1 n O A 1}$ are replaced with $\kappa_{A D 1}$ and $z_{1 n A D 1}$, respectively. Formula (8) is applied also to express $\sigma_{A D 2}$. For this purpose, $\varepsilon$ is replaced with $\varepsilon_{A D 2}$. The strain, $\varepsilon_{A D 2}$ is found by replacing of $\kappa_{O A 2}$ and $z_{1 n O A 2}$ with $\kappa_{A D 2}$ and $z_{1 n A D 2}$ in formula (23). The non-linear algebraic equations obtained by substituting of (36), (37), $\sigma_{A D 1}$ and $\sigma_{A D 2}$ in (32) - (35) are solved numerically together with 
equations (38) and (39) with respect to $\kappa_{A D 1}, z_{1 n A D 1}, \kappa_{A D 2}, z_{1 n A D 2}, M_{A D 1}$ and $M_{A D 2}$ by the MatLab computer program by applying the quasi Newton methods.

The strain energy in the internal part of beam portion $A D$ is expressed as

$$
U_{A D 2}=\int_{a_{1}}^{a_{2}}\left[\int_{0}^{R_{4}}\left(\int_{0}^{2 \pi} u_{0 A D 2} r d \varphi\right) d r\right] d x
$$

where $u_{0 A D 2}$ is obtained by replacing of $\varepsilon$ with $\varepsilon_{A D 2}$ in (9).

The strain energy in the un-cracked beam portion, $D H$, is written as

$$
U_{U N}=\int_{a_{2}}^{l}\left[\int_{0}^{R}\left(\int_{0}^{2 \pi} u_{0 U N} r d \varphi\right) d r\right] d x
$$

where the strain energy density, $u_{O U N}$ is expressed by replacing of $\varepsilon$ with $\varepsilon_{U N}$ in formula (9). The curvature and the coordinate of the neutral axis that are needed in order to obtain the distribution of strain, $\varepsilon_{U N}$, are determined by the equations of equilibrium of the stress resultants in the cross-section of the uncrack beam portion

$$
\begin{gathered}
N_{U N}=\int_{0}^{R}\left(\int_{0}^{2 \pi} \sigma_{U N} r d \varphi\right) d r \\
M_{U N}=\int_{0}^{R}\left(\int_{0}^{2 \pi} \sigma_{U N} r^{2} \sin \varphi d \varphi\right) d r
\end{gathered}
$$

where

$$
\begin{gathered}
N_{U N}=0 \\
M_{U N}=F x \cos \alpha-F R_{1} \sin \alpha
\end{gathered}
$$

The normal stress, $\sigma_{U N}$, is expressed by replacing of $\varepsilon$ with $\varepsilon_{U N}$ in (8). The distribution of $\varepsilon_{U N}$ is found by (13). For this purpose, $\kappa_{O A 1}$ and $z_{1 n O A 1}$ are replaced with $\kappa_{U N}$ and $z_{1 n U N}$, respectively. The non-linear algebraic equations obtained by substituting of (44), (45) and $\sigma_{U N}$ in (42) and (43) are solved numerically with respect to $\kappa_{U N}$ and $z_{1 n U N}$ by the MatLab computer program by applying the quasi Newton methods.

By using the integrals of Maxwell-Mohr [18], the projection of the displacement of the application point of the force, $F$, on the direction of $F$ is expressed as

$$
\begin{gathered}
w_{p r}=\int_{0}^{a_{1}} M_{O A 1}(x) \kappa_{O A 1}(x) d x+ \\
+\int_{a_{1}}^{a_{2}} M_{A D 1}(x) \kappa_{A D 1}(x) d x+
\end{gathered}
$$




$$
+\int_{a_{2}}^{l} M_{U N}(x) \kappa_{U N}(x) d x
$$

where $M_{O A 1}(x), M_{A D 1}(x)$ and $M_{U N}(x)$

are the bending moments in beam portions $O A, A D$ and $U N$ induced by the unit loading. Since

$$
\begin{aligned}
& M_{O A 1}(x)=x \cos \alpha-R_{4} \sin \alpha \\
& M_{A D 1}(x)=x \cos \alpha-R_{4} \sin \alpha \\
& M_{U N}(x)=x \cos \alpha-R_{4} \sin \alpha
\end{aligned}
$$

expression (46) takes the form

$$
\begin{gathered}
w_{p r}=\int_{0}^{a_{1}}\left(x \cos \alpha-R_{4} \sin \alpha\right) \kappa_{O A 1}(x) d x+ \\
+\int_{a_{1}}^{a_{2}}\left(x \cos \alpha-R_{4} \sin \alpha\right) \kappa_{A D 1}(x) d x+ \\
+\int_{a_{2}}^{l}\left(x \cos \alpha-R_{4} \sin \alpha\right) \kappa_{U N}(x) d x
\end{gathered}
$$

By substituting of (6), (7), (29), (30), (31), (40), (41) and (50) in (5), one obtains the following solution to the strain energy release rate:

$$
\begin{aligned}
G_{a_{1}}= & \frac{F}{2 \pi R_{3}}\left[\left(a_{1} \cos \alpha-R_{4} \sin \alpha\right) \kappa_{O A 1}\left(a_{1}\right)-\right. \\
- & \left.\left(a_{1} \cos \alpha-R_{4} \sin \alpha\right) \kappa_{A D 1}\left(a_{1}\right)\right]- \\
-\frac{1}{2 \pi R_{3}}[ & \int_{R_{4}}^{R}\left(\int_{0}^{2 \pi} u_{0 O A 1}\left(a_{1}\right) r d \varphi\right) d r+ \\
+ & \int_{R_{3}}^{R_{4}}\left(\int_{0}^{2 \pi} u_{0 O A 2}\left(a_{1}\right) r d \varphi\right) d r+ \\
+ & \int_{0}^{R_{3}}\left(\int_{0}^{2 \pi} u_{0 O A 3}\left(a_{1}\right) r d \varphi\right) d r- \\
- & \int_{R_{4}}^{R}\left(\int_{0}^{2 \pi} u_{0 A D 1}\left(a_{1}\right) r d \varphi\right) d r- \\
& \left.-\int_{0}^{R_{4}}\left(\int_{0}^{2 \pi} u_{0 A D 2}\left(a_{1}\right) r d \varphi\right) d r\right]
\end{aligned}
$$


The integration in (47) is performed by using the MatLab computer program. It should be mentioned that $R, \kappa_{O A 1}, \kappa_{A D 1}, u_{0 O A 1}, u_{0 O A 2}, u_{0 O A 3}, u_{0 A D 1}$ and $u_{0 A D 2}$ are obtained by (1), (9), (14), (15), (17), (18), (19), (20), (24), (27), (28), (32) - (35), (38) and (39) at $x=a_{1}$.

The strain energy release rate is derived also at a small increase, $\delta a_{2}$, of the length of the outer crack (Figure1). For this purpose, formula (5) is re-written as

$$
G_{a_{2}}=\frac{F}{2 \pi R_{4}} \frac{\partial w_{p r}}{\partial a_{2}}-\frac{1}{2 \pi R_{4}} \frac{\partial U}{\partial a_{2}}
$$

By substituting of (6), (7), (29), (30), (31), (40), (41) and (50) in (52), one derives

$$
\begin{aligned}
G_{a_{2}}= & \frac{F}{2 \pi R_{4}}\left[\left(a_{2} \cos \alpha-R_{4} \sin \alpha\right) \kappa_{A D 1}\left(a_{2}\right)-\right. \\
& \left.-\left(a_{2} \cos \alpha-R_{4} \sin \alpha\right) \kappa_{U N}\left(a_{2}\right)\right]- \\
- & \frac{1}{2 \pi R_{4}}\left[\int_{R_{4}}^{R}\left(\int_{0}^{2 \pi} u_{0 A D 1}\left(a_{2}\right) r d \varphi\right) d r+\right. \\
& +\int_{0}^{R_{4}}\left(\int_{0}^{2 \pi} u_{0 A D 2}\left(a_{2}\right) r d \varphi\right) d r- \\
& \left.-\int_{0}^{R}\left(\int_{0}^{2 \pi} u_{0 U N}\left(a_{2}\right) r d \varphi\right) d r\right]
\end{aligned}
$$

where $R, \kappa_{A D 1}, \kappa_{U N}, u_{0 A D 1}, u_{0 A D 2}$ and $u_{0 U N}$ are obtained by (1), (9), (14), (15), (17), (18), (19), (20), (24), (27), (28), (32) - (35), (38) and (39) at $x=a_{1}$. The integration in (53) is carried-out by the MatLab computer program.

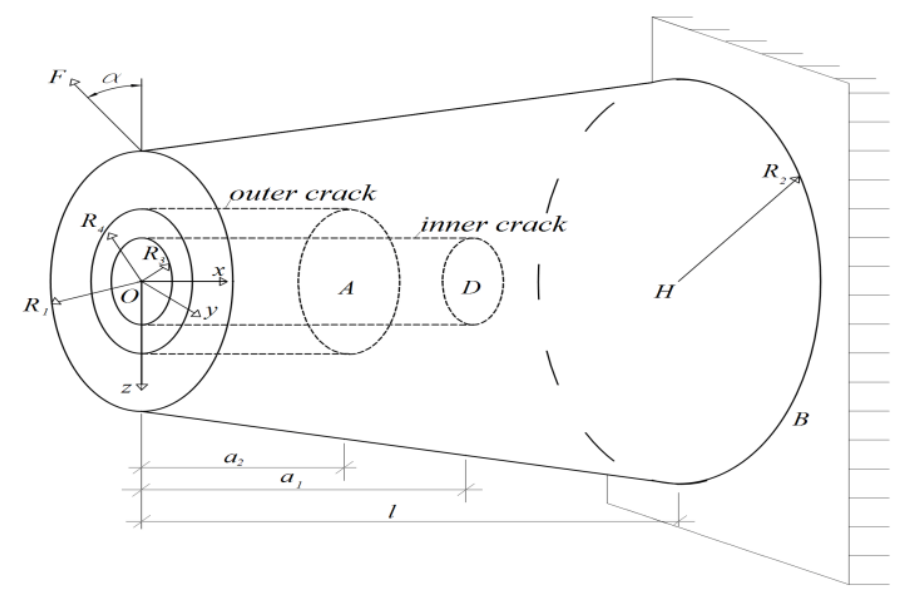

Figure 2. Geometry and loading of nonhomogeneous cantilever beam with two concentric longitudinal cracks (the inner crack is longer than the outer one).

The longitudinal fracture behaviour of the nonhomogeneous non-linear elastic cantilever beam is analyzed also for the case when the inner crack is longer than the outer one (Figure 2). First, a small increase, $\delta a_{1}$, of the length of the inner crack has been given. By considering the balance of the energy, the strain energy release rate is expressed as 


$$
\begin{aligned}
& G_{a_{1}}= \frac{F}{2 \pi R_{3}}\left[\left(a_{1} \cos \alpha-R_{4} \sin \alpha\right) \kappa_{A D 1}\left(a_{1}\right)-\right. \\
&-\left.\left(a_{1} \cos \alpha-R_{4} \sin \alpha\right) \kappa_{U N}\left(a_{1}\right)\right]- \\
&- \frac{1}{2 \pi R_{3}}\left[\int_{R_{3}}^{R}\left(\int_{0}^{2 \pi} u_{0 A D 1}\left(a_{1}\right) r d \varphi\right) d r+\right. \\
&+\int_{0}^{R_{3}}\left(\int_{0}^{2 \pi} u_{0 A D 2}\left(a_{1}\right) r d \varphi\right) d r- \\
&\left.-\int_{0}^{R}\left(\int_{0}^{2 \pi} u_{0 U N}\left(a_{1}\right) r d \varphi\right) d r\right]
\end{aligned}
$$

where the curvature, $\kappa_{A D 1}$, is determined at $x=a_{1}$ by using the equations of equilibrium of the stress resultants in the cross-sections of the external and internal parts of beam portion $A D$.

For the beam in Figure 2, these equations for equilibrium are written as

$$
\begin{gathered}
N_{A D 1}=\int_{R_{3}}^{R}\left(\int_{0}^{2 \pi} \sigma_{A D 1} r d \varphi\right) d r \\
M_{A D 1}=\int_{R_{3}}^{R}\left(\int_{0}^{2 \pi} \sigma_{A D 1} r^{2} \sin \varphi d \varphi\right) d r \\
N_{A D 2}=\int_{0}^{R_{3}}\left(\int_{0}^{2 \pi} \sigma_{A D 2} r d \varphi\right) d r \\
M_{A D 2}=\int_{0}^{R_{3}}\left(\int_{0}^{2 \pi} \sigma_{A D 2} r^{2} \sin \varphi d \varphi\right) d r
\end{gathered}
$$

The curvature, $\kappa_{U N}$, that participates in (50) is obtained at $x=a_{1}$ from the equations (42) and (43) for equilibrium of the stress resultants in the cross-section of the un-cracked beam portion.

The strain energy release rate is derived also at a small increase, $\delta a_{2}$, of the length of the outer crack in the cantilever beam configuration shown in Figure 2. By analyzing the balance of the energy, one obtains

$$
\begin{aligned}
& G_{a_{2}}= \frac{F}{2 \pi R_{4}}\left[\left(a_{2} \cos \alpha-R_{4} \sin \alpha\right) \kappa_{O A 1}\left(a_{2}\right)-\right. \\
&\left.-\left(a_{2} \cos \alpha-R_{4} \sin \alpha\right) \kappa_{A D 1}\left(a_{2}\right)\right]- \\
&-\frac{1}{2 \pi R_{4}}\left[\int_{R_{4}}^{R}\left(\int_{0}^{2 \pi} u_{0 O A 1}\left(a_{2}\right) r d \varphi\right) d r+\right. \\
&+\int_{R_{3}}^{R_{4}}\left(\int_{0}^{2 \pi} u_{0 O A 2}\left(a_{2}\right) r d \varphi\right) d r+ \\
&+\int_{0}^{R_{3}}\left(\int_{0}^{2 \pi} u_{0 O A 3}\left(a_{2}\right) r d \varphi\right) d r-
\end{aligned}
$$




$$
\begin{aligned}
& -\int_{R_{3}}^{R}\left(\int_{0}^{2 \pi} u_{0 A D 1}\left(a_{2}\right) r d \varphi\right) d r- \\
& \left.-\int_{0}^{R_{3}}\left(\int_{0}^{2 \pi} u_{0 A D 2}\left(a_{2}\right) r d \varphi\right) d r\right]
\end{aligned}
$$

where the curvature, $\kappa_{O A 1}$, is found from equations (14), (15), (17), (18), (19), (20), (24), (27) and (28) at $x=a_{2}$.

\subsection{Analysis of the complementary strain energy}

In order to verify solution (47), the strain energy release rate is derived also by applying the following formula [14]:

$$
G=\frac{d U^{*}}{d A_{c}}
$$

where $U^{*}$ is the complementary strain energy, $A_{c}$ is the crack area. Since

$$
d A_{c}=l_{c f} d a_{1}
$$

expression (56) takes the form

$$
G=\frac{d U^{*}}{l_{c f} d a_{1}}
$$

where $d a_{1}$ is an elementary increase of the length of the internal crack.

The complementary strain energy in the beam shown in Figure 1 is written as

$$
\begin{aligned}
& U^{*}=U_{O A 1}^{*}+U_{O A 2}^{*}+U_{O A 3}^{*}+ \\
& +U_{A D 1}^{*}+U_{A D 2}^{*}+U_{U N}^{*}
\end{aligned}
$$

where $U_{O A 1}^{*}, U_{O A 2}^{*}$ and $U_{O A 3}^{*}$ are the complementary strain energies in the external, interstitial and internal parts of beam portion $O A$, respectively, $U_{A D 1}^{*}$ and $U_{A D 2}^{*}$ are the complementary strain energies in the external and internal parts of beam portion $A D$, respectively, $U_{U N}^{*}$ is the complementary strain energy in the un-cracked beam portion, $D H$.

Formula (7) is applied to obtain $U_{O A 1}^{*}$. For this purpose, $u_{0 O A 1}$ is replaced with the complementary strain energy density, $u_{0 O A 1}^{*}$. The following formula is used to calculate $u_{0 O A 1}^{*}[16]$ :

$$
u_{0 O A 1}^{*}=\sigma \varepsilon-u_{0 O A 1}
$$


By substituting of (8) and (9) in (60), one derives

$$
u_{0 O A 1}^{*}=-L \varepsilon\left(1-\frac{\varepsilon}{g}\right)^{t}-\frac{L g}{t+1}\left(1-\frac{\varepsilon}{g}\right)^{t+1}+\frac{L g}{t+1}
$$

where $\varepsilon$ is found by (13).

The complementary strain energy in the interstitial part of beam portion, $O A$, is obtained by replacing of $u_{0 O A 2}$ with the complementary strain energy density, $u_{0 O A 2}^{*}$, in (29). Formula (65) is used to calculate $u_{0 O A 2}^{*}$. For this purpose, $\varepsilon$ is replaced with $\varepsilon_{O A 2}$.

Formula (30) is applied to obtain $U_{O A 3}^{*}$. For this purpose, $u_{0 O A 3}$ is replaced with the complementary strain energy density, $u_{0 O A 3}^{*}$. The complementary strain energy density is calculated by replacing of $\varepsilon$ with $\varepsilon_{O A 3}$ in (65).

The complementary strain energy in the external part of beam portion, $A D$, is found by replacing of $u_{0 A D 1}$ with the complementary strain energy density, $u_{0 A D 1}^{*}$, in (31). Formula (65) is used to obtain $u_{0 A D 1}^{*}$. For this purpose, $\varepsilon$ is replaced with $\varepsilon_{A D 1}$.

The strain energy density, $u_{0 A D 2}$, is replaced with the complementary strain energy density, $u_{0 A D 2}^{*}$, in (40) to obtain $U_{A D 2}^{*}$. The complementary strain energy density, $u_{0 A D 2}^{*}$, is found by replacing of $\varepsilon$ with $\varepsilon_{A D 2}$ in $(65)$.

The complementary strain energy in the un-cracked beam portion, $D H$, is obtained by replacing of $u_{0 U N}$ with $u_{0 U N}^{*}$ in (41). The complementary strain energy density, $u_{0 U N}^{*}$, is expressed by replacing of $\varepsilon$ with $\varepsilon_{U N}$ in (65).

By substituting of (4), $U_{O A 1}^{*}, U_{O A 2}^{*}, U_{O A 3}^{*}, U_{A D 1}^{*}, U_{A D 2}^{*}, U_{U N}^{*}$ and (61) in (62), one derives

$$
\begin{aligned}
G_{a_{1}}= & \frac{1}{2 \pi R_{3}}\left[\int_{R_{4}}^{R}\left(\int_{0}^{2 \pi} u_{0 O A 1}^{*}\left(a_{1}\right) r d \varphi\right) d r+\right. \\
& +\int_{R_{3}}^{R_{4}}\left(\int_{0}^{2 \pi} u_{0 O A 2}^{*}\left(a_{1}\right) r d \varphi\right) d r+ \\
& +\int_{0}^{R_{3}}\left(\int_{0}^{2 \pi} u_{0 O A 3}^{*}\left(a_{1}\right) r d \varphi\right) d r- \\
& -\int_{R_{4}}^{R}\left(\int_{0}^{2 \pi} u_{0 A D 1}^{*}\left(a_{1}\right) r d \varphi\right) d r- \\
& \left.-\int_{0}^{R_{4}}\left(\int_{0}^{2 \pi} u_{0 A D 2}^{*}\left(a_{1}\right) r d \varphi\right) d r\right]
\end{aligned}
$$

where $R, u_{0 O A 1}^{*}, u_{0 O A 2}^{*}, u_{0 O A 3}^{*}, u_{0 A D 1}^{*}, u_{0 A D 2}^{*}$ and $u_{0 U N}^{*}$ are obtained at $x=a_{1}$. The integration in (66) is performed using the computer program MatLab. It should be noted that the release rate of strain energy obtained by (66) is exactly the same as that obtained by (51). This fact is a 
confirmation of the solution for the strain energy release rate when increasing the length of the internal crack in the cantilever beam in Figure 1.

The strain energy release rate at increase of the length of the outer crack in the cantilever in Figure 1 is found by applying (62). For this purpose, $a_{1}$ is replaced with $a_{2}$. Besides, $l_{c f}$ is calculated as

$$
l_{c f}=2 \pi R_{4}
$$

By substituting of $U_{O A 1}^{*}, U_{O A 2}^{*}, U_{O A 3}^{*}, U_{A D 1}^{*}, U_{A D 2}^{*}, U_{U N}^{*},(63)$ and (67) in (62), one obtains

$$
\begin{aligned}
G_{a_{2}}= & \frac{1}{2 \pi R_{4}}\left[\int_{R_{4}}^{R}\left(\int_{0}^{2 \pi} u_{0 A D 1}^{*}\left(a_{2}\right) r d \varphi\right) d r+\right. \\
& +\int_{0}^{R_{4}}\left(\int_{0}^{2 \pi} u_{0 A D 2}^{*}\left(a_{2}\right) r d \varphi\right) d r- \\
& \left.-\int_{0}^{R}\left(\int_{0}^{2 \pi} u_{0 U N}^{*}\left(a_{2}\right) r d \varphi\right) d r\right]
\end{aligned}
$$

The MatLab computer program is used to perform the integration in (68). The strain energy release rate found by (68) matches exactly that obtained by (54).

Formula (62) is applied also to verify the solutions to the strain energy release rate for the cantilever beam configuration shown in Figure 2. First, an increase of the inner crack arm is considered. By using formula (62), one derives

$$
\begin{aligned}
G_{a_{1}}= & \frac{1}{2 \pi R_{3}}\left[\int_{R_{3}}^{R}\left(\int_{0}^{2 \pi} u_{0 A D 1}^{*}\left(a_{1}\right) r d \varphi\right) d r+\right. \\
& +\int_{0}^{R_{3}}\left(\int_{0}^{2 \pi} u_{0 A D 2}^{*}\left(a_{1}\right) r d \varphi\right) d r- \\
& \left.-\int_{0}^{R}\left(\int_{0}^{2 \pi} u_{0 U N}^{*}\left(a_{1}\right) r d \varphi\right) d r\right]
\end{aligned}
$$

For the case when increase of the outer crack has been given in the beam in Figure 2, the application of formula (69) leads to the following expression for the strain energy release rate:

$$
\begin{aligned}
G_{a_{2}}= & \frac{1}{2 \pi R_{4}}\left[\int_{R_{4}}^{R}\left(\int_{0}^{2 \pi} u_{0 O A 1}^{*}\left(a_{2}\right) r d \varphi\right) d r+\right. \\
& +\int_{R_{3}}^{R_{4}}\left(\int_{0}^{2 \pi} u_{0 O A 2}^{*}\left(a_{2}\right) r d \varphi\right) d r+ \\
& +\int_{0}^{R_{3}}\left(\int_{0}^{2 \pi} u_{0 O A 3}^{*}\left(a_{2}\right) r d \varphi\right) d r- \\
& -\int_{R_{3}}^{R}\left(\int_{0}^{2 \pi} u_{0 A D 1}^{*}\left(a_{2}\right) r d \varphi\right) d r-
\end{aligned}
$$




$$
\left.-\int_{0}^{R_{3}}\left(\int_{0}^{2 \pi} u_{0 A D 2}^{*}\left(a_{2}\right) r d \varphi\right) d r\right]
$$

The integration in (69) and (70) is performed by the MatLab computer program. It should be mentioned that the strain energy release rates obtained by (69) and (70) are exact matches of these found by (54) and (59). This fact is a verification of the solutions to the strain energy release rate for the crack problem shown in Figure 2.

\section{Results and discussion}

This section presents numerical results obtained by applying the solutions to the strain energy release rate derived in the previous section of the paper. It has been given that $R_{1}=0.004 \mathrm{~m}, l=0.120 \mathrm{~m}, g=0.2$, $t=1.4$ and $F=5 \mathrm{~N}$.

First, the influence of the varying radius of the beam cross-section on the longitudinal fracture behaviour of the beam is investigated. The cantilever beam configuration shown in Figure 1 is considered. The variation of the radius along the beam length is characterized by $R_{2} / R_{1}$ ratio. The strain energy release rate is presented in non-dimensional form by using the formula $G_{N}=G /\left(L_{0} R_{1}\right)$.

The influence of the varying radius of the beam cross-section along the beam length on the longitudinal fracture behaviour is illustrated in Figure 3 where the strain energy release rate in non-dimensional form is presented as a function of $R_{2} / R_{1}$ ratio at $R_{3} / R_{1}=0.2, R_{4} / R_{1}=0.7, a_{1} / l=0.3$ and $a_{2} / l=0.5$. It is evident from Figure 3 that the strain energy release rate decreases with increasing of $R_{2} / R_{1}$ ratio. One can observe also in Figure 3 that the strain energy release rate derived assuming increase of the outer crack is higher than that obtained assuming increase of the inner crack.

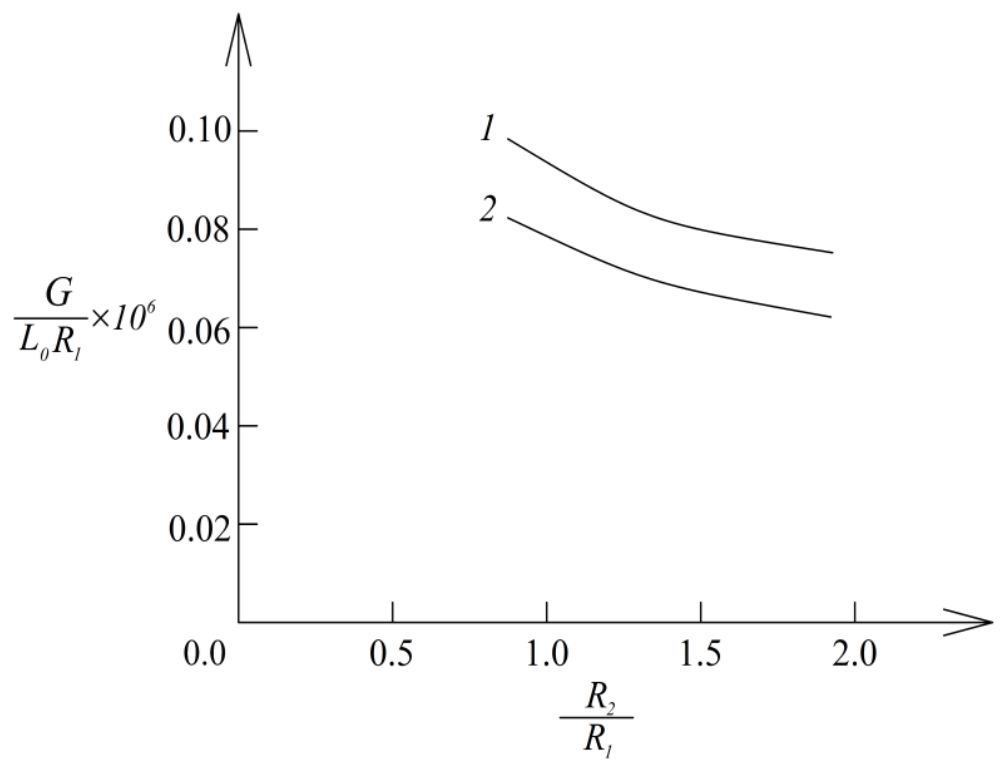

Figure 3. The strain energy release rate in non-dimensional form presented as a function of $R_{2} / R_{1}$ ratio (curve 1 -at increase of the outer crack, curve 2 - at increase of the inner crack).

The effect of material nonhomogeneity on the longitudinal fracture behaviour is analyzed. 


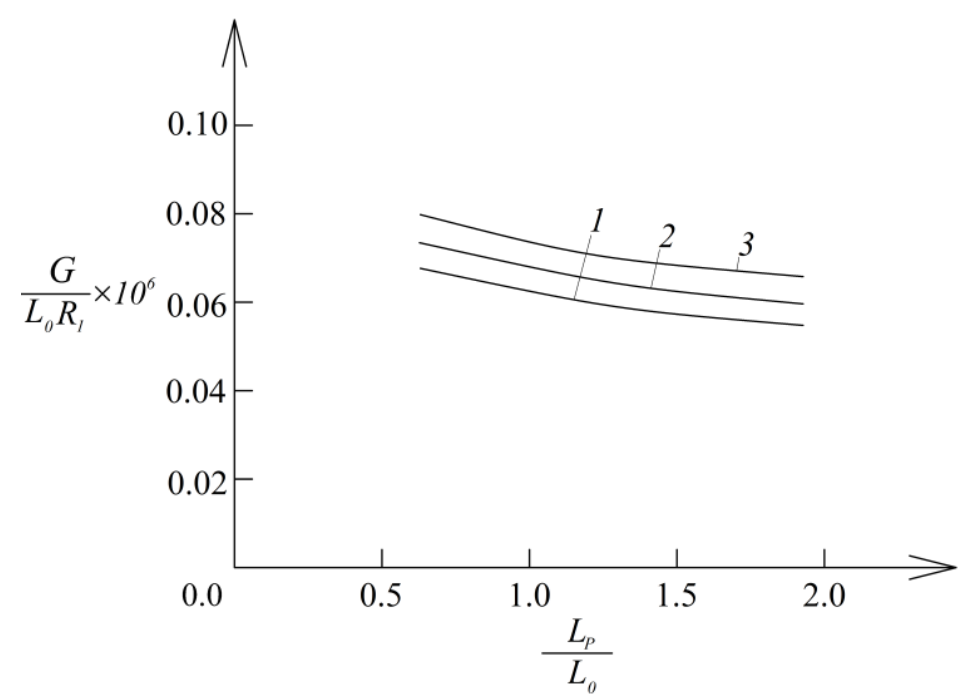

Figure 4. The strain energy release rate in non-dimensional form presented as a function of $L_{P} / L_{0}$ ratio (curve 1 - at $R_{3} / R_{1}=0.15$, curve $2-$ at $R_{3} / R_{1}=0.30$ and curve $3-$ at $R_{3} / R_{1}=0.45$ ).

The material nonhomogeneity in radial direction of the cross-section of the beam is characterized by $L_{P} / L_{0}$ ratio. The beam configuration shown in Figure 1 is under consideration. The solution to the strain energy release rate derived at increase of the inner crack is used. The location of the inner crack in radial direction is characterized by $R_{3} / R_{1}$ ratio. The strain energy release rate in non-dimensional form is presented as a function of $L_{P} / L_{0}$ ratio in Figure 4 at three $R_{3} / R_{1}$ ratios for $R_{2} / R_{1}=1.2, R_{4} / R_{1}=0.8$, $a_{1} / l=0.4$ and $a_{2} / l=0.5$. The curves in Figure 4 indicate that the strain energy release rate decreases with increasing of $L_{P} / L_{0}$ ratio. Concerning the effect of the location of the inner crack, it can be observed in Figure 4 that the strain energy release rate increases with increasing of $R_{3} / R_{1}$ ratio.

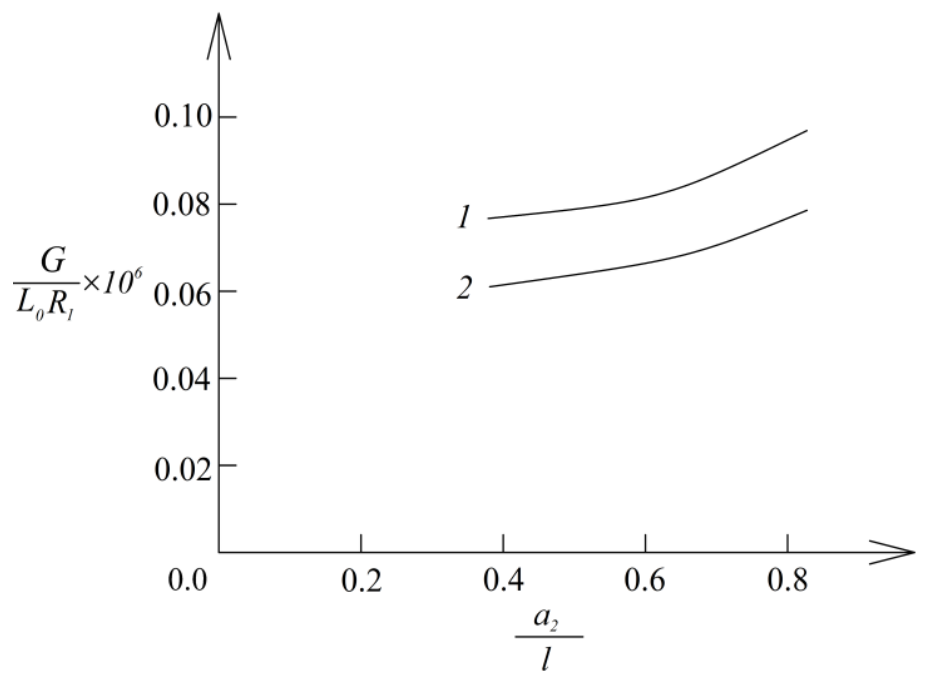

Figure 5. The strain energy release rate in non-dimensional form presented as a function of $a_{2} / l$ ratio (curve 1 -at non-linear elastic material behaviour and curve 2 - at linear-elastic material behaviour).

The influence of the crack length on the longitudinal fracture behaviour is studied. The beam configuration in Figure 1 is considered. The solution to the strain energy release rate obtained at increase of the outer crack is applied. The length of the outer crack is characterized by $a_{2} / l$ ratio. The strain energy 
release rate in non-dimensional form is presented as a function of $a_{2} / l$ ratio in Figure 5 at $a_{1} / l=0.25$, $R_{2} / R_{1}=1.2, R_{3} / R_{1}=0.3$ and $R_{4} / R_{1}=0.6$. The curves in Figure 5 show that the strain energy release rate increases with increasing of $a_{2} / l$ ratio. The effect of the non-linear mechanical behaviour of the material on the longitudinal fracture is studied too. For this purpose, the strain energy release rate in nondimensional form obtained at linear-elastic behaviour of the nonhomogeneous material is presented as function of $a_{2} / l$ ratio in Figure 5 for comparison with the non-linear elastic solution.

The linear-elastic solution to strain energy release rate is derived by substituting of $g=1$ and $t=1$ in solution (49) since at $g=1$ and $t=1$ the non-linear stress-strain relation (8) transforms in the Hooke's law assuming that $L$ is the modulus of elasticity of the nonhomogeneous material.

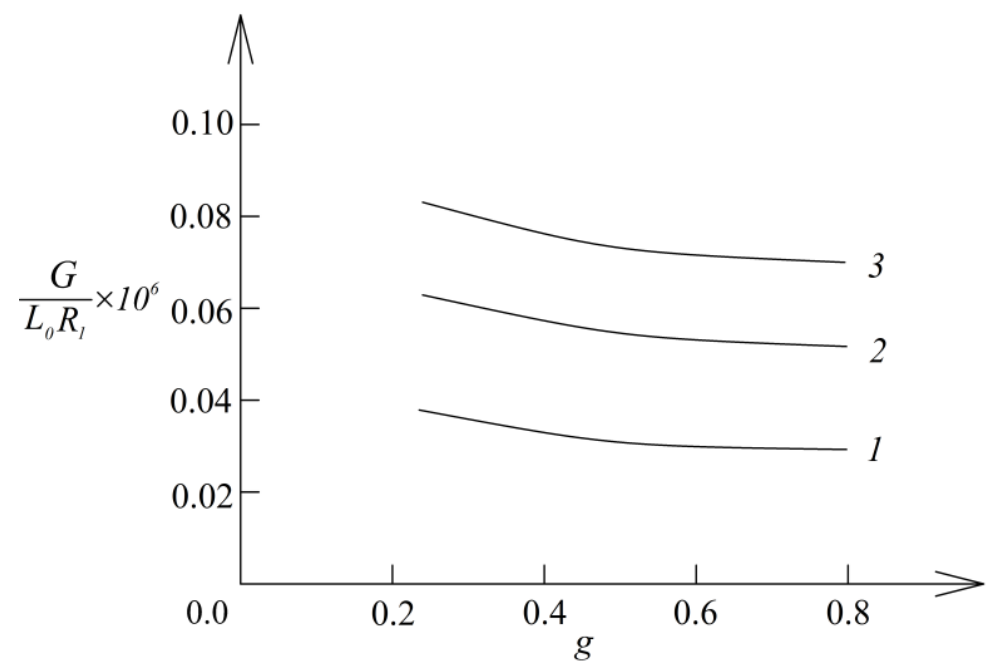

Figure 6. The strain energy release rate in non-dimensional form presented as a function of $g$ (curve 1 -at $F=3 N$, curve $2-$ at $F=4 N$ and curve $3-$ at $F=5 N$ ).

The effect of material constant, $g$, on the longitudinal fracture behaviour is evaluated. The beam configuration in Figure 1 is considered.

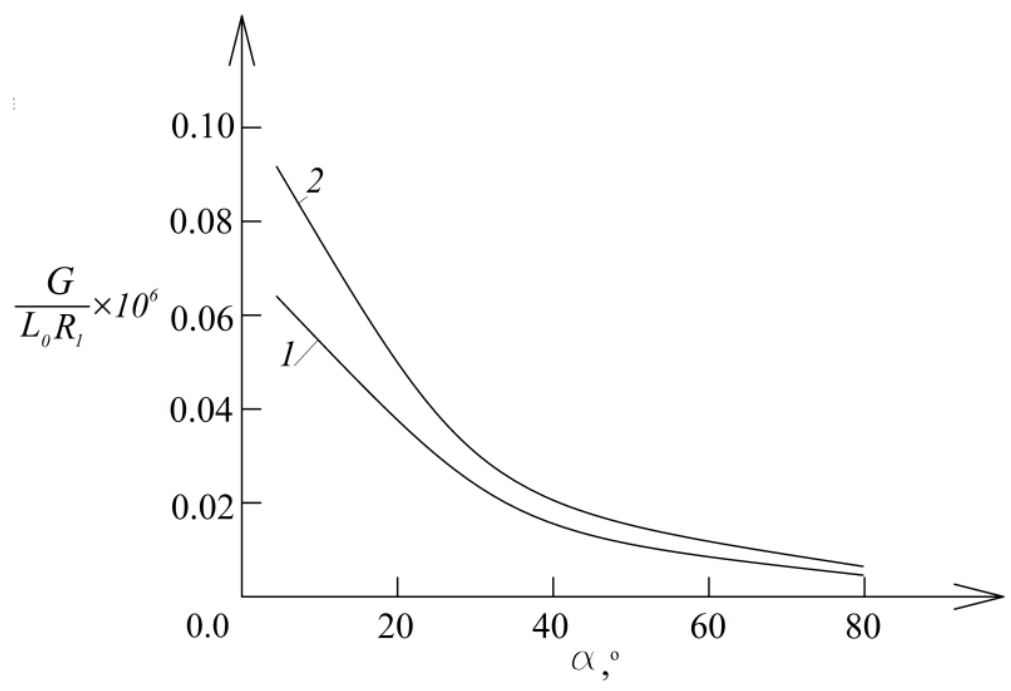

Figure 7. The strain energy release rate in non-dimensional form presented as a function of $\alpha$ (curve $1-a t$ increase of the inner crack in the beam configuration with shorter inner crack (Figure 1) and curve $2-a t$ increase of the inner crack in the beam configuration with longer inner crack (Figure 2)). 
The solution to the strain energy release rate obtained at increase of the inner crack is applied. One can get an idea about the effect of $g$ on the longitudinal fracture from Figure 6 where the strain energy release rate in non-dimensional form is presented as a function of $g$ at three values of the external force, $F$, for $a_{1} / l=0.3, a_{2} / l=0.5, R_{2} / R_{1}=0.8, R_{3} / R_{1}=0.2$ and $R_{4} / R_{1}=0.7$. The curves in Figure 6 indicate that the strain energy release rate decreases with increasing of $g$.

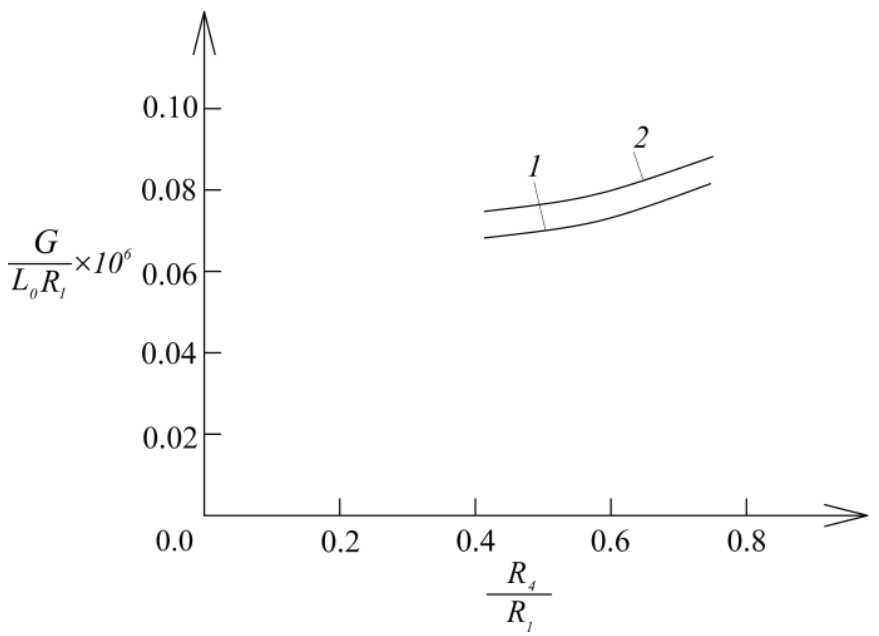

Figure 7. The strain energy release rate in non-dimensional form presented as a function of $R_{4} / R_{1}$ ratio (curve $1-$ at $a_{2} / l=0.4$ and curve $2-$ at $a_{2} / l=0.5$ ).

The influence of the angle, $\alpha$, on the longitudinal fracture behaviour is analyzed. The cantilever beam configuration with longer inner crack shown in Figure 2 is considered. The solution to the strain energy release rate derived at increase of the inner crack is applied. In order to evaluate the influence of $\alpha$ on the longitudinal fracture behaviour, the strain energy release rate in non-dimensional form is presented as a function of $\alpha$ in Figure 7 at $R_{2} / R_{1}=0.6, R_{3} / R_{1}=0.2$ and $R_{4} / R_{1}=0.7$. One can observe in Figure 7 that the strain energy release rate decreases with increasing of $\alpha$. The strain energy release rate in nondimensional form obtained at increase of the inner crack in the cantilever beam configuration with shorter inner crack (Figure 1) is presented also in Figure 7 for comparison with the strain energy release rate in the beam with longer inner crack. The curves in Figure 7 indicate that the strain energy release rate in the beam with longer inner crack is higher in comparison with that in the beam with shorter inner crack.

The influence of the location of the outer crack on the longitudinal fracture behaviour is also analyzed. For this purpose, the strain energy release rate in non-dimensional form is plotted against $R_{4} / R_{1}$ ratio in Figure 8 at two $a_{2} / l$ ratios for $a_{1} / l=0.3, R_{2} / R_{1}=1.8$ and $R_{3} / R_{1}=0.2$. The beam configuration shown in Figure 1 is considered. The solution to the strain energy release rate at increase of the outer crack is used. It is evident from Figure 8 that the strain energy release rate increases with increasing of $R_{4} / R_{1}$ ratio.

\section{Conclusions}

The fracture behaviour of an inhomogeneous cantilever beam with two concentric longitudinal cracks is studied in terms of the strain energy release rate. The beam under study has a circular cross-section. The radius of the cross section varies linearly along the length of the beam. Two concentric longitudinal cracks of different lengths are located in any radial direction. The beam has a continuously (uniformly) varying material inhomogeneity in the radial direction of the cross-section. Moreover, the material has a nonlinear elastic mechanical behaviour. A solution for the strain energy release rate is found by considering the energy balance. The fracture behaviour is also analysed considering the complementary strain energy stored in the beam to verify the solution for the strain energy release rate. The effect of varying the radius of the cross 
section along the length of the beam on the fracture behaviour is studied. For this purpose, the strain energy release rate is presented as a function of the ratio of the radius of the cross-section in the clamped end of the beam to the radius in the free end, $R_{2} / R_{1}$. It is found that the strain energy release rate decreases with increasing of $R_{2} / R_{1}$ ratio. The strain energy release rate is presented also as a function of $R_{3} / R_{1}$ ratio in order to evaluate the effect of the location of the inner crack in radial direction on the fracture behaviour. The analysis reveals that the strain energy release rate increases with increasing of $R_{3} / R_{1}$ ratio. Concerning the influence of the length of the outer crack on the fracture behaviour, it is found that the strain energy release rate increases with increasing of $a_{2} / l$ ratio. The strain energy release rate is studied also as a function of $L_{P} /$ $L_{0}$ ratio (this ratio characterizes the material nonhomogeneity along the radius of the cross-section of the beam). The investigation shows that the strain energy release rate decreases with increasing of $L_{P} / L_{0}$ ratio. The fracture behaviour of the inhomogeneous cantilever beam configuration is also studied as a function of. The study indicates that the strain energy release rate decreases with increasing size. The fracture behaviour of the beam configuration where the outer crack is longer is compared with that of the beam where the inner crack is longer. It is found that the strain energy release rate is higher when the inner crack is longer. The strain energy release rate solutions derived in the present work can be useful in the design of inhomogeneous members considering their longitudinal fracture behaviour. Moreover, the strain energy release rate solutions can be applied to check crack growth by comparing the calculated strain energy release rate with the critical rate (the latter is known as fracture toughness).

\section{References}

[1] Suresh, S., Mortensen, A.: Fundamentals of functionally graded materials. IOM Communications Ltd, London, 1998.

[2] EL-Wazery, M.S., EL-Desouky, A.R.: A review on functionally graded ceramic-metal materials, Material Environment Science, 6(2015), 2, 1369-1376.

[3] Mahamood, R.M., Akinlabi, E.T.: Functionally Graded Materials, Springer, 2017.

[4] Bohidar, S.K., Sharma, R., Mishra, P.R.: Functionally graded materials: A critical review, International Journal of Research, 1(2014), 4, 289-301.

[5] Eishen, J.W.: Fracture of non-homogeneous materials. Int J Fract, 34(1987), 1, 3-22.

[6] Erdogan, F.: Fracture mechanics of functionally graded materials, Comp. Eng., 5(1995), 2, 753-770.

[7] Ozturk, M., Erdogan, F.: Mode I crack problem in an inhomogeneous orthotropic medium, Int J Fract, 35(1997), 2, 869-883.

[8] Parameswaran, V., Shukla, A.: Dynamic fracture of a functionally gradient material having discrete property variation, J Mater Sci, 33(1998), 1, 3303-3311.

[9] Wang, B.L., Noda, N.: Thermally induced fracture of a smart functionally graded composite structure, Theor Appl Fract Mech, 35(2001), 1, 93-109.

[10] Jin, Z.H., Paulino, G.H.: Transient thermal stress analysis of an edge crack in a functionally graded material. Int J fract, 107(2001), 1, 73-98.

[11] Paulino, G.C.: Fracture in functionally graded materials, Engng Fract Mech, 69(2002), 6, 1519-1530.

[12] Tilbrook, M.T., Moon, R.J., Hoffman, M.: Crack propagation in graded composites, Composite Science and Technology, 65(2005), 6, 201-220.

[13] Carpinteri, A., Pugno, N.: Cracks in re-entrant corners in functionally graded materials, Engineering Fracture Mechanics, 73(2006), 1, 1279-1291.

[14] Rizov, V.I.: Delamination of Multilayered Functionally Graded Beams with Material Nonlinearity, International Journal of Structual Stability and Dynamics, (2018), doi.org/10.1142/SO219455418500517.

[15] Rizov, V.: An analytical solution to the strain energy release rate of a crack in functionally graded beams, European journal of mechanics A/solids, 65(2017), 6, 301-312.

[16] Rizov, V.: Lengthwise fracture analyses of functionally graded beams by the Ramberg-Osgood equation, Engineering Review, 38(2018), 3, 309-320.

[17] Rudih, O.L., Sokolov, G.P., Pahomov, V.L.: Introduction to non-linear structural mechanics. IASV, 1998.

[18] Zubchaninov, V. G.: Theory of elasticity and plasticity. Vishaia shkola. 1990. 